\title{
Asymptotic Stability of Singular Solution for Camassa-Holm Equation
}

\section{Yuetian Gao}

School of Mathematics and Computer Science, Yunnan Minzu University, Kunming, China

Email: sweetgyt@126.com

How to cite this paper: Gao, Y.T. (2021) Asymptotic Stability of Singular Solution for Camassa-Holm Equation. Journal of Applied Mathematics and Physics, 9, 1505-1514. https://doi.org/10.4236/jamp.2021.97102

Received: June 17, 2021

Accepted: July 12, 2021

Published: July 15, 2021

Copyright (c) 2021 by author(s) and Scientific Research Publishing Inc. This work is licensed under the Creative Commons Attribution International License (CC BY 4.0). http://creativecommons.org/licenses/by/4.0/

\begin{abstract}
The aim of this paper is to study singular dynamics of solutions of Camassa-Holm equation. Based on the semigroup theory of linear operators and Banach contraction mapping principle, we prove the asymptotic stability of the explicit singular solution of Camassa-Holm equation.

\section{Keywords}

Asymptotic Stability, Camassa-Holm Equation, Explicit Solution, Semigroup Theory, Banach Contraction Mapping Principle
\end{abstract}

\section{Introduction and Main Results}

\subsection{Introduction}

Consider the well-known Camassa-Holm equation as follows (see [1]):

$$
m_{t}+c_{0} u_{x}+u m_{x}+2 m u_{x}=0,
$$

where $(t, x) \in \mathbb{R}^{+} \times \mathbb{R}, \quad u=u(t, x)$ is the velocity of fluid, $m$ is the momentum given by

$$
m=m(t, x)=u(t, x)-\alpha^{2} u_{x x}(t, x),
$$

$c_{0} \in \mathbb{R}$ is the critical speed and $\alpha \in \mathbb{R}$ relates to the length scale. Thus,

$$
u_{t}-\alpha^{2} u_{t x x}+c_{0} u_{x}+3 u u_{x}=\alpha^{2}\left(2 u_{x} u_{x x}+u u_{x x x}\right) .
$$

Given the initial value as $u(0, x)=u_{0}(x)$ for $x \in \mathbb{R}$.

The Camassa-Holm equation describes unidirectional propagation of surface water waves in shallow water area. For the global well-posedness and stability of solutions, we recommend that the reader refers to [2]-[9], etc. For the wave breaking analysis, we refer the reader to [6] [10]-[15], etc. When $c_{0}=0$ and $\alpha=1$, the Camassa-Holm equation becomes to the classical Camassa-Holm eq- 
uation, which admits a bi-Hamiltonian structure [1] [5]. Moreover, the explicit peakon solution and its stability have been established in [12] [16] [17] [18] [19], etc.

Since it is rare to see the explicit stable blowup solutions of Camassa-Holm equation, in this paper, we study the stability of the explicit solution of (1.2) as follows (see [20]):

$$
\bar{u}(t, x)=-\frac{1}{3}\left(c_{0}+\frac{x}{T-t}+\frac{1}{T-t}\right),
$$

where $T>0$ is a constant.

\subsection{Main Results}

Now, we state our main result of this paper.

Theorem 1.1. Let $s>2$ be an integer and $\delta$ is a sufficiently small constant. Then the explicit solution (1.3) of the Camassa-Holm Equation (1.2) is asymptotic stable, i.e., if the initial data $u_{0}(x)$ satisfies

$$
\left\|u_{0}(x)+\frac{1}{3}\left(c_{0}+\frac{x}{T}+\frac{1}{T}\right)\right\|_{\mathbb{H}^{s+1}(\mathbb{R})} \leq \delta,
$$

then there is a solution $u(t, x)$ of (1.2) satisfying

$$
\|u(t, x)-\bar{u}(t, x)\|_{\mathbb{H}^{s}(\mathbb{R})} \leq \frac{\tilde{C}(T-t)}{\alpha^{2}(1+C \ln (T-t))}, \quad(t, x) \in(0, T) \times \mathbb{R},
$$

where $C$ and $\tilde{C}$ are positive constants that depend on $s$.

\subsection{Notations}

Denote $\mathbb{L}^{2}(\mathbb{R})=\mathbb{L}^{2}$ and $\mathbb{H}^{s}(\mathbb{R})=\mathbb{H}^{s}$ by the Lebesgue spaces and Sobolev spaces with norms $\|\cdot\|_{\mathbb{L}^{2}}$ and $\|\cdot\|_{\mathbb{H}^{s}}$, respectively. ${ }^{*}$ denotes the convolution. $[A, B]$ stands for the commutator.

\section{Proof of Theorem 1.1}

Let

$$
u(t, x)=v(t, x)+\bar{u}(t, x),
$$

be the solution of (1.2), where $\bar{u}(t, x)=-\frac{1}{3}\left(c_{0}+\frac{x}{T-t}+\frac{1}{T-t}\right)$ is the explicit solution. Substituting (2.1) into (1.2), we get

$$
\begin{aligned}
& v_{t}-\alpha^{2} v_{t x x}+\left[\frac{\alpha^{2}}{3}\left(c_{0}+\frac{x}{T-t}+\frac{1}{T-t}\right)\right] v_{x x x}+\frac{2 \alpha^{2}}{3(T-t)} v_{x x} \\
& -\left(\frac{x}{T-t}+\frac{1}{T-t}\right) v_{x}-\frac{1}{T-t} v+3 v v_{x} \\
& =\alpha^{2}\left(2 v_{x} v_{x x}+v v_{x x x}\right), \quad \forall(t, x) \in(0, T) \times \mathbb{R}
\end{aligned}
$$

with the initial condition $v(0, x)=v_{0}(x)=u_{0}(x)+\frac{1}{3}\left(c_{0}+\frac{x}{T}+\frac{1}{T}\right)$ for $x \in \mathbb{R}$. 
For the singular coefficients in (2.2), let $v(t, x)=\psi(\tau, \rho)$ by $\tau=-\ln (T-t)$ and $\rho=\frac{x}{T-t}$, then (2.2) becomes to

$$
\begin{aligned}
& \psi_{\tau}+\rho \psi_{\rho}-\alpha^{2} \mathrm{e}^{2 \tau}\left(\psi_{\tau \rho \rho}+2 \psi_{\rho \rho}+\rho \psi_{\rho \rho}\right)+\mathrm{e}^{2 \tau}\left[\frac{\alpha^{2}}{3}\left(c_{0}+\rho+\mathrm{e}^{\tau}\right)\right] \psi_{\rho \rho \rho} \\
& +\frac{2 \alpha^{2}}{3} \mathrm{e}^{2 \tau} \psi_{\rho \rho}-\left(\rho+\mathrm{e}^{\tau}\right) \psi_{\rho}-\psi+3 \psi \psi_{\rho}=\alpha^{2} \mathrm{e}^{2 \tau}\left(2 \psi_{\rho} \psi_{\rho \rho}+\psi \psi_{\rho \rho \rho}\right) .
\end{aligned}
$$

Let $\kappa=\mathrm{e}^{-\tau} \rho$ and $\bar{v}(\tau, \kappa)=\mathrm{e}^{-\tau} \psi(\tau, \rho)$. Then (2.3) becomes to

$$
\begin{aligned}
& \bar{v}_{\tau}-\alpha^{2} \bar{v}_{\tau \kappa \kappa}-\frac{\alpha^{2}}{3} \bar{v}_{\kappa \kappa}+\mathrm{e}^{-\tau}\left[\gamma+\frac{\alpha^{2}}{3}\left(c_{0}+\kappa \mathrm{e}^{\tau}+\mathrm{e}^{\tau}\right)\right] \bar{v}_{\kappa \kappa \kappa} \\
& -(\kappa+1) \bar{v}_{\kappa}+3 \overline{V V}_{\kappa}=\alpha^{2}\left(2 \bar{v}_{\kappa} \bar{v}_{\kappa \kappa}+\overline{V V}_{\kappa \kappa \kappa}\right) .
\end{aligned}
$$

Let the operator $\mathcal{A}=\left(1-\alpha^{2} \partial_{\kappa \kappa}\right)^{\frac{1}{2}}$. Since $1-\alpha^{2} \partial_{\kappa \kappa}$ admits a fundamental solution $\wp(x)=\frac{1}{2 \alpha} \mathrm{e}^{-\left|\frac{\kappa}{\alpha}\right|}$, we have $\mathcal{A}^{-2} \bar{v}=\wp(\kappa) * \bar{v}$ for all $\bar{v} \in \mathbb{L}^{2}$. Let $w(\tau, \kappa)=\bar{v}(\tau, \kappa)-\alpha^{2} \bar{v}_{\kappa \kappa}(\tau, \kappa)$, then $\bar{v}(\tau, \kappa)=\wp * w$, where $\kappa \in \mathbb{R}$. Furthermore, we have $(\rho * w)_{\kappa \kappa}=\alpha^{-2}(\rho * w-w), \bar{v}_{\kappa}=(\wp * w)_{\kappa}$ and $\bar{v}_{\kappa \kappa \kappa}=\alpha^{-2}\left((\wp * w)_{\kappa}-w_{\kappa}\right)$. Then (2.3) can be rewritten as

$$
\begin{aligned}
& w_{\tau}+\frac{1}{3} w-\mathrm{e}^{-\tau}\left[\frac{1}{3}\left(c_{0}+\mathrm{e}^{\tau} \kappa+\mathrm{e}^{\tau}\right)\right] w_{\kappa}-\frac{1}{3} \wp * w \\
& +\left\{\mathrm{e}^{-\tau}\left[\frac{1}{3}\left(c_{0}+\mathrm{e}^{\tau} \kappa+\mathrm{e}^{\tau}\right)\right]-(\kappa+1)\right\}(\wp * w)_{\kappa}+3(\wp * w)(\wp * w)_{\kappa} \\
& =2(\wp * w)_{\kappa}(\wp * w-w)+(\wp * w)\left[(\wp * w)_{\kappa}-w_{\kappa}\right]
\end{aligned}
$$

with the initial data

$$
w_{0}(\kappa)=u_{0}(x)-\alpha^{2} u_{0}^{\prime \prime}(x)+\frac{1}{3}\left(\frac{x}{T}+\frac{1}{T}+c_{0}\right),
$$

and the boundary condition

$$
\lim _{|\kappa| \rightarrow+\infty} w(\tau, \kappa)=0, \quad \lim _{|\kappa| \rightarrow+\infty} w_{\kappa}(\tau, \kappa)=0 .
$$

Before making a priori estimate of the solutions to problems (2.5)-(2.7). We recall the following commutator estimate.

Lemma 2.1 ([21]). Let $s>0$. Then it holds

$$
\left\|\left[\mathcal{A}^{s}, u\right] v\right\|_{\mathbb{L}^{2}} \leq C\left(\left\|\partial_{x} u\right\|_{\mathbb{L}^{\infty}}\left\|\mathcal{A}^{s-1} v\right\|_{\mathbb{L}^{2}}+\left\|\mathcal{A}^{s} u\right\|_{\mathbb{L}^{2}}\|v\|_{\mathbb{L}^{\infty}}\right),
$$

where $C$ is a positive constant that depends on $s$.

Now, we derive a priori estimate of the solutions for (2.5).

Lemma 2.2. Let $s>2$ and $\alpha \neq 0$. Assume that $w$ be a solution of (2.5), then

$$
\|w\|_{\mathbb{H}^{s}} \leq \frac{1}{\left\|w_{0}\right\|_{\mathbb{H}^{s}}^{-1}-C \tau},
$$

where $C$ is a positive constant depending upon $s$. 
Proof. Applying $\mathcal{A}^{s}$ to both sides of (2.5) and taking the $\mathbb{L}^{2}$-inner product with $\mathcal{A}^{s} w$, we get

$$
\begin{aligned}
& \frac{1}{2} \frac{\mathrm{d}}{\mathrm{d} \tau}\|w\|_{\mathbb{H}^{s}}^{2}+\frac{1}{3}\|w\|_{\mathbb{H}^{s}}^{2}-\frac{1}{3} \int_{\mathbb{R}} \mathcal{A}^{s} w \mathcal{A}^{s}(\wp * w) \mathrm{d} \kappa \\
& -\mathrm{e}^{-\tau} \int_{\mathbb{R}} \mathcal{A}^{s} w \mathcal{A}^{s}\left[\left(\frac{1}{3}\left(c_{0}+\mathrm{e}^{\tau} \kappa+\mathrm{e}^{\tau}\right)\right) w_{\kappa}\right] \mathrm{d} \kappa \\
& +\int_{\mathbb{R}} \mathcal{A}^{s} w \mathcal{A}^{s}\left\{\left[\mathrm{e}^{-\tau}\left(\frac{1}{3}\left(c_{0}+\mathrm{e}^{\tau} \kappa+\mathrm{e}^{\tau}\right)\right)-(\kappa+1)\right](\wp * w)_{\kappa}\right\} \mathrm{d} \kappa \\
& +3 \int_{\mathbb{R}} \mathcal{A}^{s} w \mathcal{A}^{s}\left[(\wp * w)(\wp * w)_{\kappa}\right] \mathrm{d} \kappa \\
& =2 \int_{\mathbb{R}} \mathcal{A}^{s} w \mathcal{A}^{s}\left[(\wp * w)_{\kappa}(\wp * w-w)\right] \mathrm{d} \kappa \\
& +\int_{\mathbb{R}} \mathcal{A}^{s} w \mathcal{A}^{s}\left[(\wp * w)\left((\wp * w)_{\kappa}-w_{\kappa}\right)\right] \mathrm{d} \kappa .
\end{aligned}
$$

Next, we estimate each of terms in (2.10).

$$
\begin{gathered}
-\frac{1}{3} \int_{\mathbb{R}} \mathcal{A}^{s} w \mathcal{A}^{s}(\wp * w) \mathrm{d} \kappa=-\frac{1}{3}\|w\|_{\mathbb{H}^{s-1}}^{2}, \\
-\mathrm{e}^{-\tau} \int_{\mathbb{R}} \mathcal{A}^{s} w \mathcal{A}^{s}\left[\left(\frac{1}{3}\left(c_{0}+\mathrm{e}^{\tau} \kappa+\mathrm{e}^{\tau}\right)\right) w_{\kappa}\right] \mathrm{d} \kappa \\
\left.=\mathrm{e}^{-\tau} \int_{\mathbb{R}}\left[\left(\frac{1}{3}\left(c_{0}+\mathrm{e}^{\tau} \kappa+\mathrm{e}^{\tau}\right)\right) \mathcal{A}^{2 s} w\right]\right]_{\kappa} \mathrm{d} \kappa \\
=\frac{1}{3} \int_{\mathbb{R}} \mathcal{A}^{2 s} w \cdot w \mathrm{~d} \kappa-\frac{1}{2} \times \frac{1}{3} \int_{\mathbb{R}}\left(\mathcal{A}^{s} w\right)^{2} \mathrm{~d} \kappa=\frac{1}{6}\|w\|_{\mathbb{H}^{s}}^{2}, \\
\int_{\mathbb{R}} \mathcal{A}^{s} w \mathcal{A}^{s}\left\{\left[\mathrm{e}^{-\tau}\left(\frac{1}{3}\left(c_{0}+\mathrm{e}^{\tau} \kappa+\mathrm{e}^{\tau}\right)\right)-(\kappa+1)\right](\wp * w)_{\kappa}\right\} \mathrm{d} \kappa \\
=-\int_{\mathbb{R}}\left\{\mathcal{A}^{2 s} w\left[\mathrm{e}^{-\tau}\left(\frac{1}{3}\left(c_{0}+\mathrm{e}^{\tau} \kappa+\mathrm{e}^{\tau}\right)\right)-(\kappa+1)\right]\right\} \int_{\kappa}(\wp * w) \mathrm{d} \kappa \\
=-\int_{\mathbb{R}} \mathcal{A}^{2 s} w_{\kappa}\left[\mathrm{e}^{-\tau}\left(\frac{1}{3}\left(c_{0}+\mathrm{e}^{\tau} \kappa+\mathrm{e}^{\tau}\right)\right)-(\kappa+1)\right](\wp * w) \mathrm{d} \kappa \\
-\left(\frac{1}{3}-1\right) \int_{\mathbb{R}} \mathcal{A}^{2 s} w(\wp * w) \mathrm{d} \kappa \\
=\frac{1}{2} \times\left(\frac{1}{3}-1\right) \int_{\mathbb{R}}\left(\mathcal{A}^{s-1} w\right)^{2} \mathrm{~d} \kappa+\frac{2}{3} \int_{\mathbb{R}} \mathcal{A}^{2 s} w(\wp * w) \mathrm{d} \kappa=\frac{1}{3}\|w\|_{\mathbb{H}^{s-1}}^{2}, \\
3 \int_{\mathbb{R}} \mathcal{A}^{s} w \mathcal{A}^{s}\left((\wp * w)(\wp * w)_{\kappa}\right) \mathrm{d} \kappa \\
=-\frac{3}{2} \int_{\mathbb{R}} w_{\kappa} \mathcal{A}^{2 s}\left((\wp * w)^{2}\right) \mathrm{d} \kappa \leq \frac{3}{2}\left\|w_{\kappa}\right\|_{\mathbb{L}^{\infty}}\|w\|_{\mathbb{H}^{s-1}}^{2} \leq \frac{3}{2}\|w\|_{\mathbb{H}^{s-1}}^{3},
\end{gathered}
$$

In addition, using (2.8), we have

$$
\begin{aligned}
& 2\left|\int_{\mathbb{R}} \mathcal{A}^{s} w \mathcal{A}^{s}\left((\wp * w)_{\kappa}(\wp * w-w)\right) \mathrm{d} \kappa\right| \\
& =2\left|\int_{\mathbb{R}}\left[\mathcal{A}^{s},(\wp * w-w)\right](\wp * w)_{\kappa} \mathcal{A}^{s} w \mathrm{~d} \kappa\right| \\
& +2\left|\int_{\mathbb{R}}(\wp * w-w) \mathcal{A}^{s}(\wp * w)_{\kappa} \mathcal{A}^{s} w \mathrm{~d} \kappa\right| \\
& \leq C\left(\|\wp * w-w\|_{\mathbb{L}^{\infty}}\left\|\mathcal{A}^{s-1}(\wp * w)_{\kappa}\right\|_{\mathbb{L}^{2}}+\left\|\mathcal{A}^{s}(\wp * w-w)\right\|_{\mathbb{L}^{2}}\left\|(\wp * w)_{\kappa}\right\|_{\mathbb{L}^{\infty}}\right)\|w\|_{\mathbb{H}^{s}} \\
& +2\left(\|(\wp * w-w)\|_{\mathbb{L}^{\infty}}+\left\|(\wp * w-w)_{\kappa}\right\|_{\mathbb{L}^{\infty}}\right)\|w\|_{\mathbb{H}^{2}}^{2} \\
& \leq C\|w\|_{\mathbb{H}^{s}}^{3},
\end{aligned}
$$


similarly,

$$
\left|\int_{\mathbb{R}} \mathcal{A}^{s} w \mathcal{A}^{s}\left[(\wp * w)\left((\wp * w)_{\kappa}-w_{\kappa}\right)\right] \mathrm{d} \kappa\right| \leq C\|w\|_{\mathbb{H}^{s}}^{3},
$$

where $C$ is a positive constant depending upon $s$.

Substituting (2.11)-(2.16) into (2.10), we get $\frac{1}{2} \frac{\mathrm{d}}{\mathrm{d} \tau}\|w\|_{\mathbb{H}^{s}}^{2} \leq C\|w\|_{\mathbb{H}^{s}}^{3}$, and then $-\frac{\mathrm{d}}{\mathrm{d} \tau}\|w\|_{\mathbb{H}^{s}}^{-1} \leq C$. Integrating this inequality above with respect to $\tau$ from 0 to $\tau$, we get

$$
\|w\|_{\mathbb{H}^{s}} \leq \frac{1}{\left\|w_{0}\right\|_{\mathbb{H}^{s}}^{-1}-C \tau}
$$

This completes the proof of Lemma 2.2.

Proof of Theorem 1.1. Now, we study the well-posedness for (2.5)-(2.7). Define the linear operator $L$ as

$$
\begin{aligned}
L[w]= & -\frac{1}{3} w+\frac{1}{3} \wp * w+\mathrm{e}^{-\tau}\left[\frac{1}{3}\left(c_{0}+\mathrm{e}^{\tau} \kappa+\mathrm{e}^{\tau}\right)\right] w_{\kappa} \\
& -\left[\mathrm{e}^{-\tau}\left(\frac{1}{3}\left(c_{0}+\mathrm{e}^{\tau} \kappa+\mathrm{e}^{\tau}\right)\right)-(\kappa+1)\right](\wp * w)_{\kappa},
\end{aligned}
$$

then (2.5) becomes to

$$
w_{t}=L[w]+f(w)
$$

where $f$ is the nonlinear terms:

$$
\begin{aligned}
f(w)= & -3(\wp * w)(\wp * w)_{\kappa}+2(\wp * w)(\wp * w-w) \\
& +(\wp * w)\left[(\wp * w)_{\kappa}-w_{\kappa}\right] .
\end{aligned}
$$

Lemma 2.3. Let $s>2$. Then

- $L[w] \in \mathbb{H}^{s}$ for $\forall w \in \mathcal{D}(L)$.

- $L$ is a closed and densely defined linear operator in $\mathbb{H}^{s}$.

Proof. It is a direct verification by the definition of $L$.

Lemma 2.4. Let $s>2$. Then $L$ is a dissipative operator in $\mathbb{H}^{s}$, i.e., $(L[w], w)_{s} \leq 0$.

Proof. Using (2.11)-(2.14), a direct calculation shows that

$$
\begin{aligned}
\int_{\mathbb{R}}\left(\mathcal{A}^{s} L[w]\right) \mathcal{A}^{s} w \mathrm{~d} \kappa & =-\frac{1}{3}\|w\|_{\mathbb{H}^{s}}^{2}+\frac{1}{3}\|w\|_{\mathbb{H}^{s-1}}^{2}-\frac{1}{6}\|w\|_{\mathbb{H}^{s}}^{2}-\frac{1}{3}\|w\|_{\mathbb{H}^{s-1}}^{2} \\
& =-\frac{1}{2}\|w\|_{\mathbb{H}^{s}}^{2} \leq 0 .
\end{aligned}
$$

This completes the proof.

Lemma 2.5 (Young inequality with $\varepsilon$, see [22]). Let $a, b>0$ and $\varepsilon>0$. If $p, q \in(1, \infty)$ satisfy $\frac{1}{p}+\frac{1}{q}=1$. Then

$$
a b \leq \varepsilon a^{p}+C(\varepsilon) b^{q},
$$

where $C(\varepsilon)=(\varepsilon p)^{-\frac{q}{p}} q^{-1}$. 
Lemma 2.6. Let $s>2$. Then the operator $L$ is invertible in $\mathbb{H}^{s}$. Furthermore, it generates a $\mathbb{C}_{0}$-semigroup $(\mathbf{S}(t))_{\tau \geq 0}$ in $\mathbb{H}^{s}$.

Proof. Firstly, we show that the existence of $L^{-1}$. Indeed, we need to prove $L$ is injective and surjective. On the one hand, let $w \in \mathcal{D}(L)$ such that $L[w]=0$, then

$$
\int_{\mathbb{R}} \mathcal{A}^{s} L[w] \mathcal{A}^{s} w \mathrm{~d} \kappa=-\frac{1}{2}\|w\|_{\mathbb{H}^{s}}^{2}=0 .
$$

This combining with the boundary condition (2.7) gives that $w=0$. So the operator $L$ is injective. On the other hand, for all $g \in \mathbb{H}^{1}$, put

$$
L[w]=g .
$$

Applying $\mathcal{A}^{s}$ to (2.24) and multiplying the result by $\mathcal{A}^{s} w$, and then integrating over $\mathbb{R}$, we get

$$
\|w\|_{\mathbb{H}^{s}}^{2}=-2 \int_{\mathbb{R}} \mathcal{A}^{s} g \mathcal{A}^{s} w \mathrm{~d} \kappa .
$$

It follows from the Young inequality with $\varepsilon$ in Lemma 2.5 that

$$
\|w\|_{\mathbb{H}^{s}} \leq C\|g\|_{\mathbb{H}^{s}} .
$$

Note that $s>2$, then by the standard theory of elliptic equations (see [22]), there exists a unique weak solution $w \in \mathbb{H}^{1}$, moreover, we have $w \in \mathbb{H}^{s+1}$ if $g \in \mathbb{H}^{s}$. Thus, the operator $L$ is surjective. Secondly, by the Lumer-Phillips theorem (see [23]), the operator $L$ generates a $\mathbb{C}_{0}$-semigroup $(\mathbf{S}(t))_{\tau \geq 0}$ in $\mathbb{H}^{s}$. This completes the proof.

As a consequence, we have

Proposition 2.7. Let $s>2$. Then the Cauchy problem

$$
\left\{\begin{array}{l}
\frac{\mathrm{d}}{\mathrm{d} \tau} w=L w, \\
w(0)=w_{0}
\end{array}\right.
$$

with zero boundary condition exists a unique solution $w(\tau)=\mathbf{S}(\tau) w_{0}$, where $w_{0}$ is the initial data defined in (2.6).

Using the Duhamel's principle, the solutions of (2.19) satisfies the integral equation:

$$
w(\tau)=\mathbf{S}(\tau) w_{0}+\int_{0}^{\tau} \boldsymbol{S}(\tau-s) f(w(s)) \mathrm{d} s .
$$

To show this integral equation exists a solution, we define the solution space as

$$
B_{\delta}=\left\{w \in \mathbb{H}^{s}:\|w\|_{\mathbb{H}^{s}}<\delta \ll 1\right\},
$$

and the map $\mathcal{T}$ as

$$
\mathcal{T} w(\tau)=\mathbf{S}(\tau) w_{0}+\int_{0}^{\tau} \mathbf{S}(\tau-s) f(w(s)) \mathrm{d} s .
$$

We need to prove that $\mathcal{T}$ has a fixed point in the space $B_{\delta}$.

Lemma 2.8 ([21]). Let $s>2$. Then $B_{\delta}$ is an algebra, and 


$$
\|u v\|_{\mathbb{H}^{s}} \leq C\left(\|u\|_{\mathbb{L}^{\infty}}\|v\|_{\mathbb{H}^{s}}+\|u\|_{\mathbb{H}^{s}}\|v\|_{\mathbb{L}^{\infty}}\right),
$$

where $C$ is a positive constant depending upon $s$.

Lemma 2.9. Let $s>2$ be an integer. Assume that $\left\|w_{0}\right\|_{\mathbb{H}^{s+1}}<\delta$ for some sufficiently small $\delta>0$. Then $\mathcal{T}$ is a self-mapping on $B_{\delta}$. Moreover, $\mathcal{T}$ is a contraction mapping.

Proof. By Lemma 2.8, we have

$$
\begin{aligned}
\|f(w)\|_{\mathbb{H}^{s}} \leq & 3\left\|(\wp * w)(\wp * w)_{\kappa}\right\|_{\mathbb{H}^{s}}+2\left\|(\wp * w)_{\kappa}(\wp * w-w)\right\|_{\mathbb{H}^{s}} \\
& +\left\|(\wp * w)\left((\wp * w)_{\kappa}-w_{\kappa}\right)\right\|_{\mathbb{H}^{s}} \\
\leq & C_{1}\left(\|\wp * w\|_{\mathbb{H}^{s}}\left\|(\wp * w)_{\kappa}\right\|_{L_{L^{\infty}}}+\left\|(\wp * w)_{\kappa}\right\|_{L^{\infty}}\|(\wp * w-w)\|_{\mathbb{H}^{s}}\right. \\
& \left.+\|\wp * w\|_{\mathbb{H}^{s}}\left\|(\wp * w)_{\kappa}-w_{\kappa}\right\|_{\mathbb{L}^{\infty}}\right),
\end{aligned}
$$

where $C_{1}$ is a positive constant.

Note that $\mathbb{H}^{s} \subset \mathbb{L}^{\infty}$ and $w=\mathcal{A}^{2}(\wp(\kappa) * \bar{v})$, then using Lemma 2.2 , we have

$$
\|f(w)\|_{\mathbb{H}^{s}} \leq C_{1}\|w\|_{\mathbb{H}^{s}}^{2}<\frac{C_{1}}{\|w\|_{\mathbb{H}^{s}}^{-1}-C \tau}<\frac{C_{1}}{\delta^{-1}-C \tau}<\delta
$$

for sufficiently small $\delta$. Thus, $\mathcal{T}$ is a self-mapping on $B_{\delta}$.

To show $\mathcal{T}$ is a contraction mapping, we choose $w, \bar{w} \in B_{\delta}$, by Lemma 2.8 and a direct calculation show that

$$
\begin{aligned}
\| & f(w)-f(\bar{w}) \|_{\mathbb{H}^{s}} \\
= & \|-3(\wp * w)(\wp * w)_{\kappa}+2(\wp * w)_{\kappa}(\wp * w) \\
& -2(\wp * w)_{\kappa} w+(\wp * w)\left((\wp * w)_{\kappa}-w_{\kappa}\right) \\
& +3(\wp * \bar{w})(\wp * \bar{w})_{\kappa}-2(\wp * \bar{w})_{\kappa}(\wp * \bar{w}) \\
& +2(\wp * \bar{w})_{\kappa} \bar{w}-(\wp * \bar{w})\left((\wp * \bar{w})_{\kappa}-\bar{w}_{\kappa}\right) \|_{\mathbb{H}^{s}} \\
\leq & \| 3\left\{(\wp * \bar{w})[\wp *(\bar{w}-w)]_{\kappa}+(\wp * \bar{w})_{\kappa}[\wp *(\bar{w}-w)]\right\} \\
& +2\left\{(\wp * w)_{\kappa}[\wp *(\bar{w}-w)]+(\wp * \bar{w})[\wp *(\bar{w}-w)]_{\kappa}\right\} \\
& +3\left\{(\wp * \bar{w})[\wp *(\bar{w}-w)]_{\kappa}+(\wp * \bar{w})_{\kappa}[\wp *(\bar{w}-w)]\right\} \\
& +\left\{(\wp * \bar{w})[\wp *(\bar{w}-w)]_{\kappa}+(\wp * \bar{w})_{\kappa}[\wp *(\bar{w}-w)]\right\} \\
& +\left\{[\wp *(\bar{w}-w)] \overline{w_{K}}+(\wp * w)(\bar{w}-w)_{\kappa}\right\} \|_{\mathbb{H}^{s}} \\
\leq & C \delta\|w-\bar{w}\|_{\mathbb{H}^{s}} .
\end{aligned}
$$

Thus,

$$
\left\|\mathcal{T}_{w}(\tau)-\mathcal{T}_{\bar{w}}(\tau)\right\|_{\mathbb{H}^{s}} \leq C \delta\|w-\bar{w}\|_{\mathbb{H}^{s}} .
$$

Since $\delta>0$ is sufficiently small, $\mathcal{T}$ is a contraction mapping.

Thus, we have the following existence results.

Proposition 2.10. Let $\mathrm{s}>2$ be a fixed integer and $\delta>0$ is a sufficiently small constant. Then

- if $\left\|w_{0}\right\|_{\mathbb{H}^{s+1}}<\delta$, there exists a unique solution $w \in B_{\delta}$ to (2.5) with the ini- 
tial data (2.6) and the boundary condition (2.7).

- there exists a global solution $\psi(\tau, \rho) \in \mathbb{H}^{s}$ to (2.3) with the initial data (2.6) and the boundary condition (2.7). Moreover, if the initial data $\psi_{0}$ satisfies $\left\|\psi_{0}\right\|_{\mathbb{H}^{s+1}}<\delta$, then

$$
\|\psi\|_{\mathbb{H}^{s}} \leq \frac{\tilde{C}}{\alpha^{2} \mathrm{e}^{\tau}(1-C \tau)} .
$$

Here $C$ and $\tilde{C}$ are two positive constants that depend on $s$.

Proof. By Lemma 2.9 and the Banach fixed point theorem, the map $\mathcal{T}$ has a fixed point in $B_{\delta}$, which is a solution of Equation (2.5). Thus, there exists a global solution of (2.3) as

$$
\psi(\tau, \rho)=\mathrm{e}^{\tau} \bar{v}\left(\tau, \mathrm{e}^{-\tau} \rho\right)=\mathrm{e}^{\tau}\left((\wp * w)\left(\tau, \mathrm{e}^{-\tau} \rho\right)\right) .
$$

Furthermore, we have

$$
v_{\rho \rho}=\psi_{\rho \rho}=(\wp * w)_{\kappa \kappa} \mathrm{e}^{-\tau}=\alpha^{-2} \mathrm{e}^{-\tau}(\wp * w-w) .
$$

Thus, by Lemma 2.2, we get

$$
\begin{aligned}
\left\|\psi_{\rho \rho}\right\|_{\mathbb{H}^{s-2}} & \leq \alpha^{-2} \mathrm{e}^{-\tau}\|\wp * w-w\|_{\mathbb{H}^{s-2}} \leq \tilde{C} \alpha^{-2} \mathrm{e}^{-\tau}\|w\|_{\mathbb{H}^{s-2}} \\
& \leq \frac{\tilde{C}}{\alpha^{2} \mathrm{e}^{\tau}\left(\left\|w_{0}\right\|_{\mathbb{H}^{s}}^{-1}-C \tau\right)} \leq \frac{\tilde{C}}{\alpha^{2} \mathrm{e}^{\tau}(1-C \tau)},
\end{aligned}
$$

where we have used $\delta<1$ in the last inequality. This completes the proof.

As a consequence, we obtain that the global well-posedness of the initial value problem (2.2). This implies that the asymptotic stability of the explicit singular solution (1.3) for the Camassa-Holm Equation (1.2). Hence, we complete the proof of Theorem 1.1 .

\section{Conclusion}

In this paper, the Semigroup theory of linear operators has been used to study the asymptotic stability of the explicit blowup solution of Camassa-Holm equation. This result shows that the explicit solution is a meaningful physical solution. However, this explicit solution does not depend on the wavelength (i.e., it does not depend on $\alpha$ ). Thus, further studies are needed to construct the explicit solutions that depend on $\alpha$, and then prove their stability.

\section{Conflicts of Interest}

The author declares no conflicts of interest regarding the publication of this paper.

\section{References}

[1] Camassa, R. and Holm, D.D. (1993) An Integrable Shallow Water Equation with Peaked Solitons. Physical Review Letters, 71, 1661-1664.

https://doi.org/10.1103/PhysRevLett.71.1661

[2] Bressan, A. and Constantin, A. (2007) Global Conservative Solutions of the Camassa-Holm Equation. Archive for Rational Mechanics and Analysis, 183, 215-239. 
https://doi.org/10.1007/s00205-006-0010-Z

[3] Bressan, A. and Constantin, A. (2007) Global Dissipative Solutions of the Camassa-Holm Equation. Analysis and Applications, 5, 1-27. https://doi.org/10.1142/S0219530507000857

[4] Constantin, A. (1997) On the Cauchy Problem for the Periodic Camassa-Holm Equation. Journal of Differential Equations, 141, 218-235. https://doi.org/10.1006/jdeq.1997.3333

[5] Constantin, A. (1997) The Hamiltonian Structure of the Camassa-Holm Equation. Expositiones Mathematicae, 15, 53-85.

[6] Constantin, A. and Escher, J. (1998) Global Existence and Blow-up for a Shallow Water Equation. Annali della Scuola Normale Superiore di Pisa, Classe di Scienze, 26, 303-328.

[7] Constantin, A. (2001) On the Scattering Problem for the Camassa-Holm Equation. Proceedings of the Royal Society A: Mathematical, Physical and Engineering Sciences, 457, 953-970. https://doi.org/10.1098/rspa.2000.0701

[8] Constantin, A. and Molinet, L. (2000) Global Weak Solutions for a Shallow Water Equation. Communications in Mathematical Physics, 211, 45-61. https://doi.org/10.1007/s002200050801

[9] Constantin, A. and Lannes, D. (2009) The Hydrodynamical Relevance of the Camassa-Holm and Degasperis-Procesi Equations. Archive for Rational Mechanics and Analysis, 192, 165-186. https://doi.org/10.1007/s00205-008-0128-2

[10] Bendahmane, M., Coclite, G.M. and Karlsen, K.H. (2006) $H^{1}$-Perturbations of Smooth Solutions for a Weakly Dissipative Hyperelastic-Rod Wave Equation. $\mathrm{Me}$ diterranean Journal of Mathematics, 3, 419-432. https://doi.org/10.1007/s00009-006-0088-4

[11] Brandolese, L. (2014) Local-in-Space Criteria for Blowup in Shallow Water and Dispersive Rod Equations. Communications in Mathematical Physics, 330, 401-414. https://doi.org/10.1007/s00220-014-1958-4

[12] Constantin, A. (2000) Existence of Permanent and Breaking Waves for a Shallow Water Equation: A Geometric Approach. Annales de P Institut Fourier, 50, 321-362. https://doi.org/10.5802/aif.1757

[13] Constantin, A. and Escher, J. (1998) Wave Breaking for Nonlinear Nonlocal Shallow Water Equations. Acta Mathematica, 181, 229-243. https://doi.org/10.1007/BF02392586

[14] Liu, Y. (2006) Global Existence and Blow-Up Solutions for a Nonlinear Shallow Water Equation. Mathematische Annalen, 335, 717-735.

https://doi.org/10.1007/s00208-006-0768-1

[15] McKean, H.P. (2004) Breakdown of the Camassa-Holm Equation. Communications on Pure and Applied Mathematics, 57, 416-418. https://doi.org/10.1002/cpa.20003

[16] Constantin, A. and Strauss, W. (2002) Stability of the Camassa-Holm Solitons. Journal of Nonlinear Science, 12, 415-422. https://doi.org/10.1007/s00332-002-0517-x

[17] Constantin, A. and Molinet, L. (2001) Orbital Stability of Solitary Waves for a Shallow Water Equation. Physica D: Nonlinear Phenomena, 157, 75-89. https://doi.org/10.1016/S0167-2789(01)00298-6

[18] Constantin, A. and Strauss, W. (2000) Stability of a Class of Solitary Waves in Compressible Elastic Rods. Physics Letters A, 270, 140-148. https://doi.org/10.1016/S0375-9601(00)00255-3

[19] Hakkaev, S. and Kirchev, K. (2005) Local Well-Posedness and Orbital Stability of 
Solitary Wave Solutions for the Generalized Camassa-Holm Equation. Communications in Partial Differential Equations, 30, 761-781.

https://doi.org/10.1081/PDE-200059284

[20] Gao, Y. and Chen, J. (2021) Asymptotic Stability of Singular Waves for Dullin-Gottwald-Holm Equation. (Submitted)

[21] Kato, T. (1975) Quasi-Linear Equations of Evolution, with Applications to Partial Differential Equations. In: Everitt, W.N., Ed., Spectral Theory and Differential Equations, Vol. 448, Springer, Berlin, Heidelberg, 25-70. https://doi.org/10.1007/BFb0067080

[22] Evans, L.C. (1998) Partial Differential Equations (Graduate Studies in Mathematics, Vol. 19). American Mathematical Society, Providence, xviii+662 p.

[23] Pazy, A. (1983) Semigroups of Linear Operators and Applications to Partial Differential Equations. Vol. 44. Springer-Verlag, New York, viii+279 p. https://doi.org/10.1007/978-1-4612-5561-1 